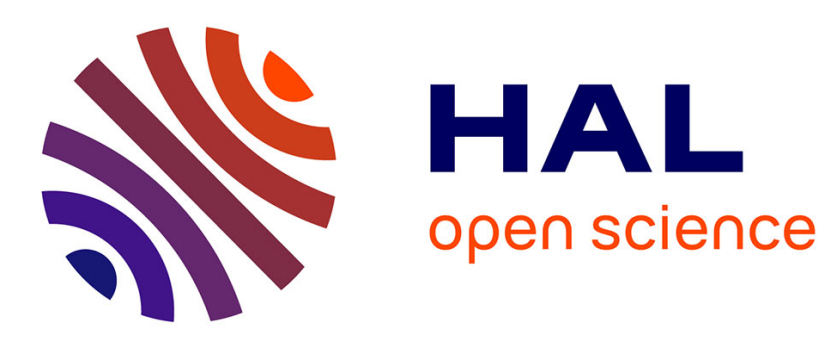

\title{
Real time Discrete Wavelet Transform architecture for self mixing interferometry signal processing
}

\author{
Syed Shahzad Hussain, Usman Zabit, Olivier Bernal
}

\section{To cite this version:}

Syed Shahzad Hussain, Usman Zabit, Olivier Bernal. Real time Discrete Wavelet Transform architecture for self mixing interferometry signal processing. 14th International Bhurban Conference on Applied Sciences and Technology (IBCAST 2017), Jan 2017, Islamabad, Pakistan. 10.1109/IBCAST.2017.7868072 . hal-01985303

\section{HAL Id: hal-01985303 https://hal.science/hal-01985303}

Submitted on 24 Jan 2020

HAL is a multi-disciplinary open access archive for the deposit and dissemination of scientific research documents, whether they are published or not. The documents may come from teaching and research institutions in France or abroad, or from public or private research centers.
L'archive ouverte pluridisciplinaire HAL, est destinée au dépôt et à la diffusion de documents scientifiques de niveau recherche, publiés ou non, émanant des établissements d'enseignement et de recherche français ou étrangers, des laboratoires publics ou privés. 


\section{Real Time Discrete Wavelet Transform Architecture for Self Mixing Interferometry Signal Processing}

\author{
Syed Shahzad Hussain, Usman Zabit \\ Faculty of Engineering \& Applied Sciences \\ Riphah International University \\ Islamabad, Pakistan \\ shahzad.hussain@riphah.edu.pk \\ usman.zabit@riphah.edu.pk
}

\author{
Olivier D Bernal \\ CNRS, LAAS, 7 avenue du colonel Roche \\ Univ de Toulouse, INPT, LAAS \\ Toulouse, France \\ obernal@laas.fr
}

\begin{abstract}
This paper describes the hardware implementation of forward discrete wavelet transform for self-mixing interferometry (SMI) signal processing. SMI signals are observed when the laser beam is backscattered after striking the target object and enter into the laser cavity thereby mixing with generated optical beam. The resulting SMI signal carries information about the remote target, so in order to retrieve the information (distance, displacement, velocity and vibration etc), SMI signal needs to be processed. Under stationary conditions, conventional Fourier transform can be used but when the conditions become non-stationary, wavelet transform based processing becomes a suitable choice. In this paper, we propose the forward wavelet architecture for real time processing of SMI signals so that target displacement can be measured with a precision of $\lambda / 10$, where $\lambda$ is the laser wavelength. The purpose of this research work is to implement the DWT based SMI signal processing algorithm in hardware so that real time displacement measurements can be retrieved from SMI signal with high precision. The proposed architecture comprises of eight processing elements using pipeline technique, where each processing element (PE) consists of two sub blocks, FIR filtering and Down sampler. The filter coefficients are pre-calculated and stored in the ROM. Simulation have been done on Xilinx ISIM® using Verilog HDL. Implementation is done on Spartan 3A FPGA, XC3S1400 (device), FG484 (package), -5 (grade speed). After implementation the synthesis results show that our proposed design is capable of operating at $605.76 \mathrm{MHz}$ with minimum clock period of 1.651ns. Our design consumes 6651 slices, 2062 slice flip flops and 11232 4-input Lookup tables. The FPGA based algorithm implementation makes the SMI sensor more attractive, as it potentially allows to execute all signal processing by embedding it on the same board resulting in a fast, real-time and autonomous SM sensor.
\end{abstract}

Keywords - Self mixing, optical feedback interferometry, wavelet transform, FPGA.

\section{INTRODUCTION}

Self-mixing interferometry (SMI) or optical feedback interferometry (OFI) technique [1-2] has attracted significant interest of sensor developers during the last two decades with applications such as measurement of displacement [3], distance, speed [4], vibrations [5] and Ablation depth [6]. A SMI system is much simpler than conventional interferometers because many optical elements such as the beam splitter, reference mirror and external photo-detector are not required. Thus, with simply constructed optical system, smart laser sensing systems have been developed using SMI [1-2]. Fig. 1 shows the target vibration or motion and its corresponding SMI signal for different $\mathrm{C}$ values.
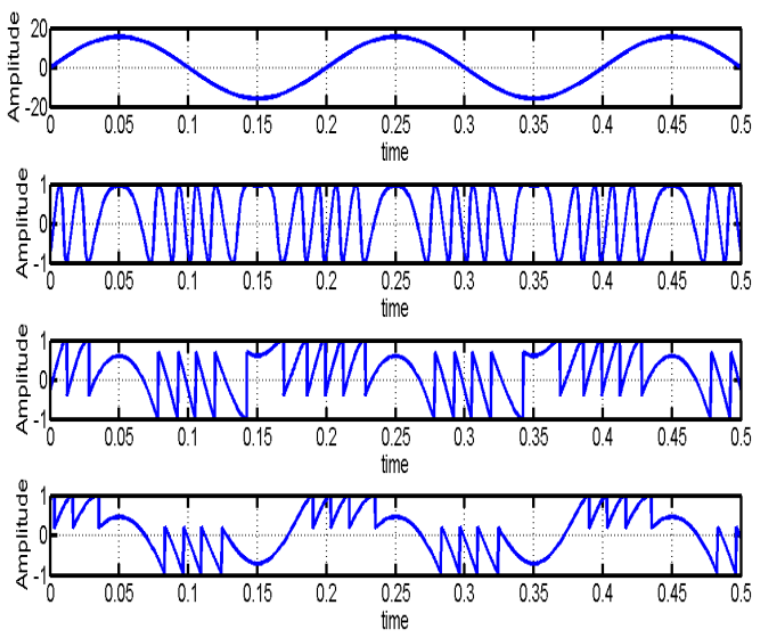

Fig. 1 (a) Target Vibration, (b) SMI signal with $\mathrm{C}=0.5$, (c) $\mathrm{SMI}$ signal with $\mathrm{C}=2.5$, (d) $\mathrm{SMI}$ signal with $\mathrm{C}=4.5$

In order to retrieve the target displacement from SMI signal with high precision, various phase unwrapping methods have been proposed in literature [7-12]. These methods differ from one another in the way laser feedback phase is interpreted and processed by them, usually involving the joint-estimation of key SMI parameters such as the optical feedback coupling factor $\mathrm{C}$ and linewidth enhancement factor $\alpha$ [7].

In [12], a Fast Fourier Transform (FFT) based approach was proposed circumventing the need of time-consuming jointestimation of these parameters. This results in fast and precise retrieval of target vibrations. However, as FFT only provides the frequency content of the signal and cannot provide timelocalisation information, so in case of time-varying nature of target motion, FFT based SMI processing becomes inadequate. Thus, the method proposed in [12] is only applicable for a restrictive/specific application of vibration sensing, valid only for stationary signals. In case of non-stationary signals, the use of Discrete Wavelet Transform (DWT), however, can solve 
the problem as DWT belongs to that area of signal processing routines specifically designed to address non-stationary signals by allowing time-frequency localisation. In literature, various authors have applied DWT based algorithms directly on the SMI signals [13-15]. In this paper, we are proposing an FPGA based DWT architecture for real time processing of SMI signals so that all such Wavelets can be effectively implemented.

Rest of the paper is organized as follows: section II describes the self-mixing theory. Section III summarizes the Discrete Wavelet Transform. Section IV elaborates the proposed architecture of DWT. Section V includes the implementation results followed by Conclusion.

\section{SELF MIXING THEORY}

SMI signals are observed, when the laser beam is backscattered after striking the target object and enters in to the optical cavity causing a mixing of generated and back scattered beam within the active laser cavity. The mixing changes the properties of laser including modulation of optical output power (OOP) of laser as a function of target motion. The variation in the OOP of the laser diode $P(t)$ caused by this optical feedback can be written as [2]

$$
P(t)=\mathrm{P}_{0}\left[1+m \cos \left(x_{F}(\mathrm{t})\right)\right]
$$

Where $\mathrm{P}_{0}$ is the emitted power under free running conditions, $m$ is the modulation index and $x_{F}(t)$ is the laser output phase in the presence of feedback, given by [2]

$$
x_{F}(t)=2 \pi \cdot \frac{D(t)}{\lambda_{F}(t) / 2}
$$

Where $\mathrm{D}(\mathrm{t})$ is the target displacement.

The emission wavelength subject to feedback $\lambda_{F}(t)$ is given by the phase equation [2]

$$
x_{o}(t)-x_{F}(t)-C \sin \left[x_{F}(t)+\arctan (\alpha)\right]=0
$$

where $x_{o}(t)$ is the laser output phase in the absence of feedback, given by

$$
x_{o}(t)=2 \pi \cdot \frac{D(t)}{\lambda_{o} / 2}
$$

where $\lambda_{0}$ is the emission wavelength under free running conditions. The feedback coupling factor $C$ is given by

$$
C=\frac{\tau_{D}}{\tau_{L}} \gamma \sqrt{1+\alpha^{2}} k_{e x t}
$$

Where $\tau_{L}$ and $\tau_{D}$ are the round trip times in the internal and external cavities respectively, $\gamma$ is the coupling efficiency, and $\mathrm{k}_{\text {ext }}$ depends linearly on the surface reflectivity of the target. $C$ plays an important role as change in the value of $\mathrm{C}$ fundamentally affects the shape of the SMI signal. $C$ factor is thus usually used to distinguish between the three optical feedback regimes and are shown in TABLE I.

TABLE I. OPTICAL FEEDBACK REGIMES

\begin{tabular}{|c|c|c|}
\hline Sr.No & C Factor & $\begin{array}{c}\text { Feedback } \\
\text { Regime }\end{array}$ \\
\hline 1 & $\mathrm{C}<1$ & Weak \\
\hline 2 & $1<\mathrm{C}<4.6$ & Moderate \\
\hline 3 & $\mathrm{C}>4.6$ & Strong \\
\hline
\end{tabular}

Usually, if optical conditions allow it then moderate feedback SMI regime [16] is much preferred over other feedback regimes. It is even possible to experimentally maintain it as well [10]. The preference accorded to moderate regime is due to the fact that its saw-tooth like fringes (see Fig. 1) can be easily detected [7]. Furthermore, these fringes also exhibit a local quasi-linearity with target motion, an aspect utilized for the sake of fast displacement extraction [8]. However, in case of speckle, above advantages are lost and significant efforts are required either to avoid its occurrence [17] or to retrieve target motion in its presence [18].

\section{DISCRETE WAVELET TRANSFORM}

Wavelet transform is widely used to investigate the time and frequency contents of a signal. As wavelet transform reliably processes such signals whose frequency contents change with time, so wavelet transform becomes a strong candidate to process the signals belonging to non-stationary conditions. The wavelets are small waves and localized in both time and frequency domain [19]. The continuous time wavelet transform of a signal $f(t)$ is given by

$$
W_{f}(s, u)=\frac{1}{\sqrt{s}} \int_{-\infty}^{\infty} f(t) \psi^{*}\left(\frac{t-u}{s}\right) d t
$$

Where $\mathrm{a}$ and $\mathrm{b}$ are scaling and translation parameters. From (6), the discrete wavelet transform becomes

$$
w(n)=\sum_{k=-\infty}^{\infty} x(n) * h(2 n-k)
$$

As seen in (7), the DWT passes the given signal to multiple filters followed by decimation. In general, DWT decomposes the input signal in to approximate (cA) and detailed coefficients (cD). An approximate coefficient represents the low frequency components in the signal while the detailed coefficients represent the high frequency components present in the signal. This process will be achieved by passing the same input signal through low and high pass filters simultaneously. By convolving input signal with low pass filter coefficients, the approximate coefficients will be obtained while convolving the same input signal with high pass filter coefficients will result in the detailed coefficients. The outputs of both filters are then down sampled by 2. Fig. 2 shows the single level decomposition structure of DWT. 


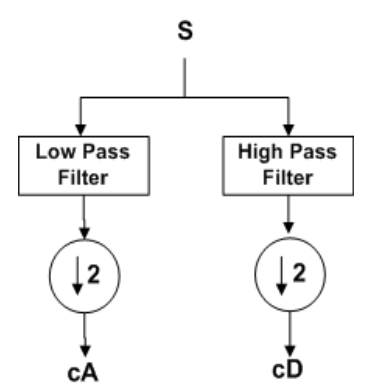

Fig. 2 DWT single level decomposition structure

\section{PROPOSED ARCHITECTURE}

For real time processing of SMI related signals, we have proposed a forward DWT architecture, detailed below. As DWT decomposes the input signal in to approximate and detailed coefficients, so this process will be achieved by convolving the input signal with both low pass and high pass filter coefficients. As explained previously, a single level DWT decomposition involves two steps, 1) FIR filtering and 2) down-sampling by 2 .

The architecture of single level DWT decomposition called single processing element (PE) and is shown in Fig. 3.

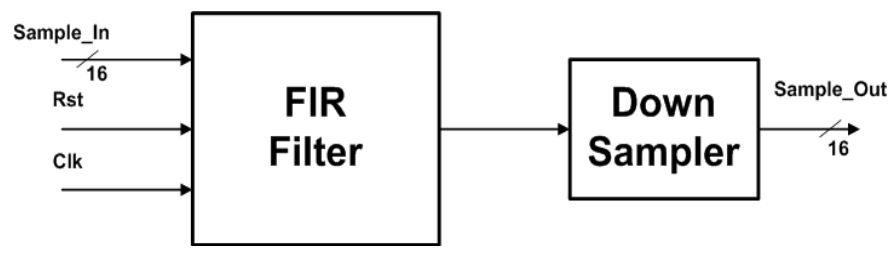

Fig. 3 Single Processing Element (PE)

The FIR filter coefficients are pre-calculated and stored in a read only memory (ROM). On every clock cycle, FIR filter takes the 16-bit new sample and produces the 16-bit output sample. Whenever a new sample arrives at the input, it will be stored in the first delay line register and the previous sample will be shifted to the second delay line register and so on until the last delay line register is updated. The length of delay line register file depends upon the filter order and is equal to $\mathrm{N}-1$, where $\mathrm{N}$ is the filter order. The FIR filter architecture is shown in Fig. 4.

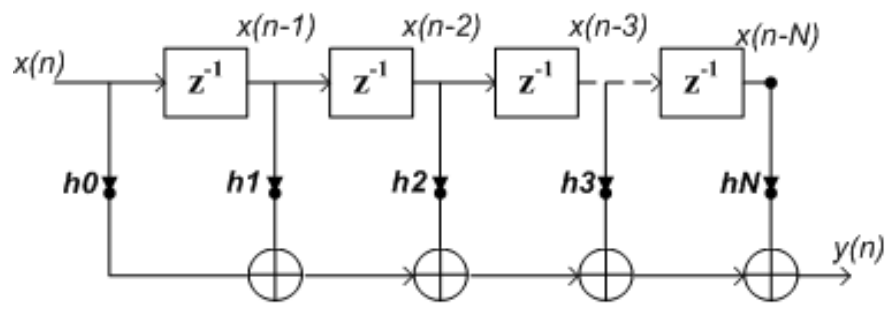

Fig. 4 FIR Filter Architecture
The second step of DWT is down sampling. Down sampling of a signal is given by

$$
y(n)=x(n M)
$$

Where $\mathbf{M}$ is a positive integer.

By using (8), sampling rate can be reduced by a factor of $\mathrm{M}$. In DWT, down-sampling by 2 is required so in this case $\mathrm{M}=$ 2. Down sampling by 2 is achieved by inserting down sampler module immediately after the FIR filter. The output of FIR filter becomes the input to down sampler. The down sampler retains every odd sample and discards the even samples. This process is achieved by slowing down the clock of down sampler block. The down sampler block is thus running at half clock rate as compared to FIR filter block clock rate. As we are using Daubechies based DWT, with decomposition level 8 and scaling level 8, so our proposed architecture comprises of eight Processing Elements (PE1-PE8). Each PE takes the 16bit input sample and gives the 16-bit output sample. The output of first PE (PE1) will become the input of second PE (PE2) and the process continues til PE8. The proposed pipelined forward DWT architecture is shown in Fig. 5.

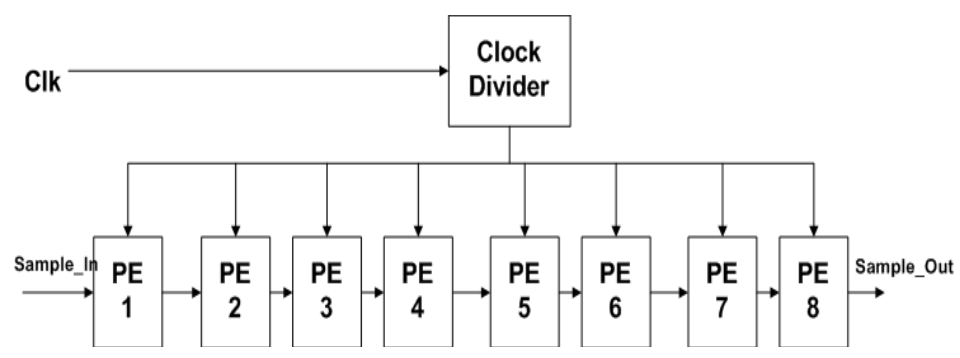

Fig. 5 Proposed DWT Architecture

\section{IMPLEMENTATION RESULTS}

The proposed architecture is implemented using Verilog HDL and Xilinx ISE 14.5 Web pack®. Simulation is done in Xilinx ISIM®. Fig. 6 and 7 shows the simulation results of FIR filter and down sampler.

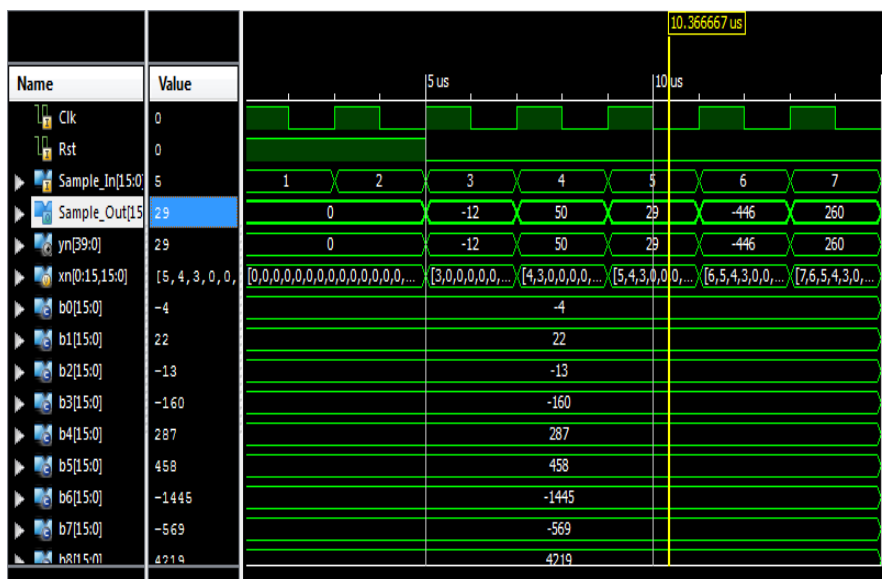

Fig. 6 Simulation Results of FIR Filter 
In Fig 6, initially Rst signal is high, so FIR filter module did not read any sample on input. The output of the FIR filter becomes zero until Rst signal is high. After two clock cycles Rst goes low and in $3^{\text {rd }}$ clock cycle FIR reads the first sample and stores it in first delay line register $\mathrm{xn}(0)$ and also produces first output sample in the same clock cycle. In the next clock cycle FIR filter reads the new input sample and update its delay line registers. In Fig 6, xn is delay line register file containing 16 registers and b0-b15 are filter coefficients.
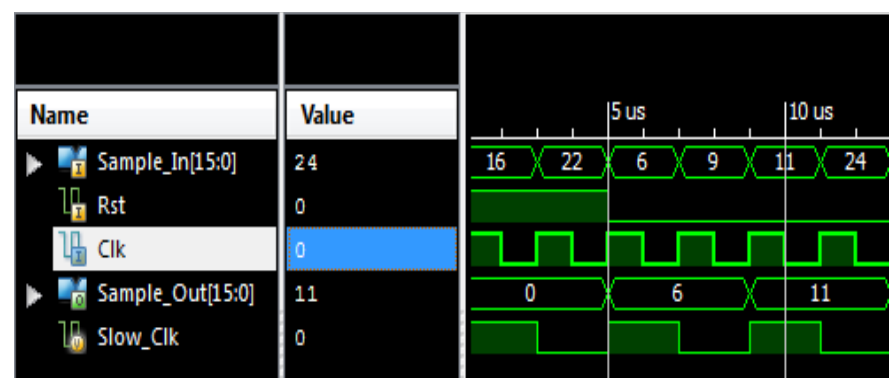

Fig. 7 Simulation Results of Down Sampler

Simulation results of down sampler block are shown in Fig. 7. During the first two clock cycles due to active high Rst signal, no sample will be read at input. The down sampler reads the new sample on every cycle of Clk but writes the output sample on every cycle of Slow_Clk. From Fig. 7 it can seen that every second sample will be dropped. The proposed architecture is implemented on Spartan 3A FPGA, XC3S1400 device, FG 484 package, -5 grade speed and RTL schematics are shown in Fig. 8 and 9.

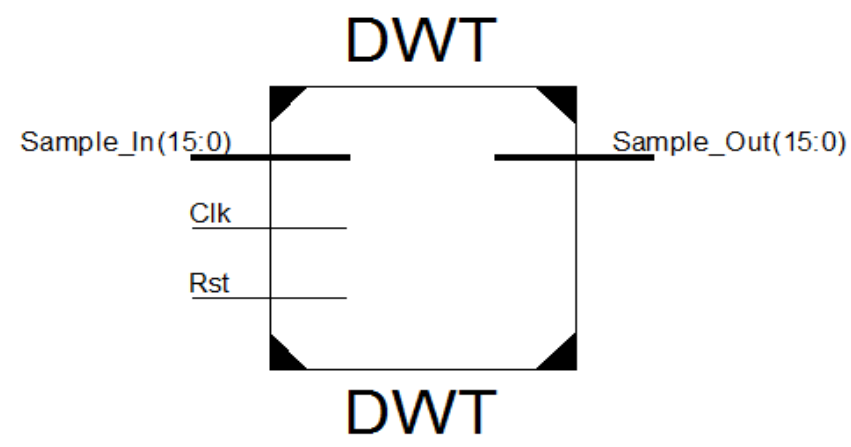

Fig. 8 DWT Top Module

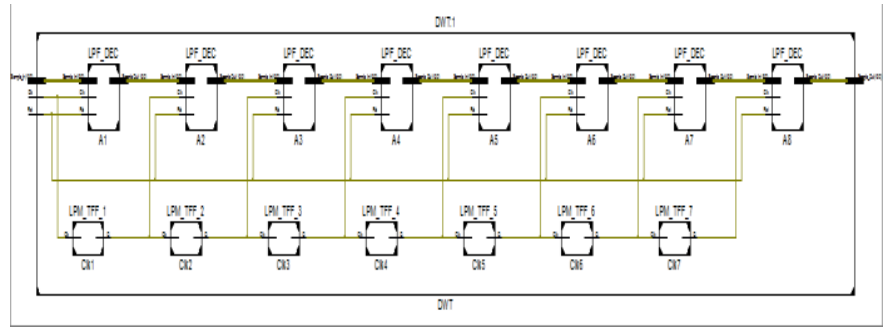

Fig. 9 RTL Schematic of Proposed Architecture
In Fig. 9 the LPF module corresponds to single processing element (see Fig. 3) which contains two blocks, FIR and down sampler block. Similarly the LPM module in Fig. 9 is just a 1bit counter which will be incremented on every tick of clock and is used to slow down the system clock. The inside view of LPM module is shown in Fig. 10.

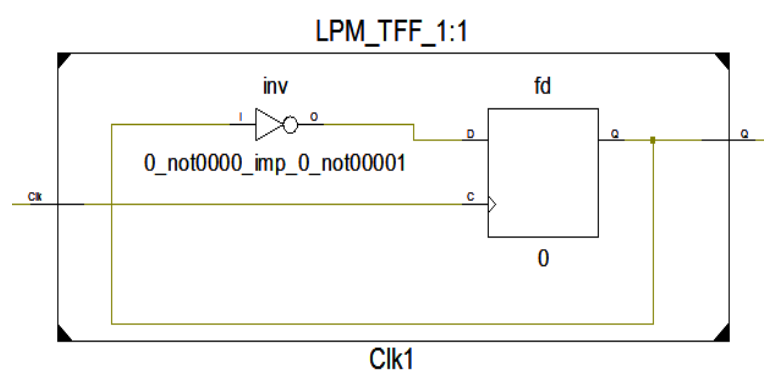

Fig. 10 inside the LPM module

The synthesis results of proposed architecture are shown in TABLE II.

TABLE II. SYNTHESIS RESULTS

\begin{tabular}{|c|c|}
\hline Slices & 6651 \\
\hline Slice Flip Flops & 2062 \\
\hline 4-input LUT & 11232 \\
\hline Bounded IOB,s & 34 \\
\hline MULT 18X18 & 32 \\
\hline Maximum Frequency (MHz) & 605.7 \\
\hline
\end{tabular}

Our proposed architecture is capable of operating at 605.76 $\mathrm{MHz}$ with minimum clock period of $1.61 \mathrm{~ns}$. From TABLE II, our design can achieve the maximum throughput of $9.93 \mathrm{Gbps}$ on the cost of 6651 slices, 2062 slice flip flops, 11232 look up tables and 32 Multipliers.

\section{CONCLUSION}

We have proposed the forward Discrete Wavelet Transform architecture for real time processing of SM signals to extract target vibration or motion with high precision. The proposed architecture comprises of eight processing elements and each processing element implements single level DWT decomposition. To achieve high throughput, the proposed architecture is implemented using pipeline technique. Furthermore, synthesis results show that our proposed design is capable of operating at $605.76 \mathrm{MHz}$ with minimum clock period of $1.651 \mathrm{~ns}$ and achieves 9.9 Gbps throughput.

\section{REFERENCES}

[1] T. Taimre, M. Nikoli'c, K. Bertling, Y. L. Lim, T. Bosch, and A. D. Rakic, "Laser feedback interferometry: a tutorial on the self-mixing effect for coherent sensing", Advances in Optics and Photonics, vol. 7, no. 3 , pp. 570-631, 2015. 
[2] S. Donati, "Developing self-mixing interferometry for instrumentation and measurements," Laser \& Photonics Review, vol. 6, pp. 393-417, May 2012.

[3] U. Zabit, O. D. Bernal, and T. Bosch, "Design and analysis of an embedded accelerometer coupled self-mixing laser displacement sensor", IEEE Sensors Journal, vol.13, no.6, pp. 2200-2207, 2013.

[4] Norgia, Michele, D. Melchionni, and A. Pesatori, "Self-mixing instrument for simultaneous distance and speed measurement", Optics and Lasers in Engineering , 2016.

[5] Z. A. Khan, U. Zabit, O. D. Bernal, M. O. Ullah, and T. Bosch, "Adaptive Cancellation of Parasitic Vibrations Affecting a Self-Mixing Interferometric Laser Sensor", IEEE Trans. on Instrumentation \& Measurements, vol. x, pp. -, doi: 10.1109/TIM.2016.2626018 (accepted), 2016.

[6] A. G. Demir, P. Colombo, M. Norgia, and B. Previtali "Evaluation of Self-Mixing Interferometry Performance in the Measurement of Ablation Depth", IEEE Transactions on Instrumentation and Measurement, vol.65, no.11, pp. 2621-2630, 2016.

[7] C. Bes, G. Plantier, and T. Bosch, "Displacement measurements using a self-mixing laser diode under moderate feedback," Instrumentation and Measurement, IEEE Transactions on, vol. 55, no. 4, pp. 1101-1105, Aug 2006.(3)

[8] U. Zabit, O. D. Bernal, and T. Bosch, "Self-mixing Sensor for Real-time Measurement of Harmonic and Arbitrary Displacements", IEEE International Instrumentation and Measurement Technology Conference, I2MTC, pp 754-758, Graz, Austria, 13-16 May 2012.(4)

[9] Y. Fan, Y. Yu, J. Xi, and J. F. Chicharo, "Improving the measurement performance for a self-mixing interferometry-based displacement sensing system," Applied Optics, vol. 50, no. 26, pp. 5064-5072, 2011.(5)

[10] O. Bernal, U. Zabit, and T. Bosch, "Robust method of stabilization of optical feedback regime by using adaptive optics for a self-mixing microinterferometer laser displacement sensor," Selected Topics in Quantum Electronics, IEEE Journal of, vol. 21, no. 4, pp. 1-8, July 2015.

[11] A. L. Arriaga, F. Bony, and T. Bosch, "Real-time algorithm for versatile displacement sensors based on self-mixing interferometry," Sensors Journal, IEEE, vol. 16, no. 1, pp. 195-202, 2016.

[12] U. Zabit, O. D. Bernal, and T. Bosch, "Time-Frequency signal processing for a self-mixing laser sensor for vibration measurement", IEEE International SENSORS Conference, Taipei, Taiwan, 28-31 October 2012.

[13] Y. Sun, Y. Yu \& J. Xi, "Wavelet transform based de-noising method for self mixing interferometry signals," 3rd Asia Pacific Optical Sensors Conference, Sydney, Jan 31-Feb 03,2012.

[14] O. D. Bernal, H. C. Seat, U. Zabit, F. Surre and T. Bosch, "Robust Detection of Non Regular Interferometric Fringes from a Self-Mixing Displacement Sensor using Bi-Wavelet Transform," IEEE Sensors Journal, vol. 16, no 22, pp 7903-7910, 2016.

[15] A. Jha, F. J. Azcona, C. Yanez and S. Royo, "Extraction of Vibration Parameters from Optical Feedback Interferometry signals using wavelets," Applied Optics, vol. 54, no. 34, pp. 10106-10113, 2015.

[16] O. D. Bernal, U. Zabit, and T. Bosch, "Classification of laser selfmixing interferometric signal under moderate feedback," Applied Optics, vol. 53, no. 4, pp. 702-708, 2014.

[17] R. Atashkhooei, S. Royo, F. Azcona, and U. Zabit, "Analysis and control of speckle effects in self-mixing interferometry," 2011 IEEE SENSORS Proceedings, 2011.

[18] U. Zabit, O. D. Bernal, and T. Bosch, "Self-Mixing Laser Sensor for Large Displacements: Signal Recovery in the Presence of Speckle", IEEE Sensors Journal, vol. 13, no. 2, pp. 824-831, 2012.

[19] K. P. Soman, N. G. Resmi and K. I. Ramachandran, Insight into Wavelets: From Theory to Practice, $3^{\text {rd }}$ edition, PHI Learning, 2010. 